\title{
Intermittent hypoxia promotes melanoma lung metastasis via oxidative stress and inflammation responses in a mouse model of obstructive sleep apnea
}

Lian $\mathrm{Li}^{1+}$, Fangyuan Ren ${ }^{1 \dagger}$, Chao Qi ${ }^{2}$, Leiqian Xu', Yinshan Fang ${ }^{2}$, Maoli Liang ${ }^{1}$, Jing Feng ${ }^{1}$, Baoyuan Chen ${ }^{1}$, Wen Ning ${ }^{2^{*}}$ and Jie Cao ${ }^{1 *}$

\begin{abstract}
Background: Recently, increased tumor incidence and cancer-related mortality have been reported among patients with obstructive sleep apnea (OSA). Intermittent hypoxia $(\mathrm{IH})$, the hallmark feature of OSA, contributes to the metastasis of tumors. However, the molecular mechanisms by which tumor metastasis is accelerated by OSA-like IH remain to be elucidated.

Methods: C57BL/6 J male mice were subjected to intravenous injection of B16F10 melanoma cells before receiving $\mathrm{IH}$ treatment. Then, the animals were randomly distributed into three groups $(n=8$ each): normoxia $(\mathrm{N})$ group, IH group, and antioxidant tempol group ( $\mathrm{HTT}$, exposed to $\mathrm{IH}$ after treatment with tempol). After the mice were sacrificed, the number and weight of lung metastatic colonies were assessed. The lung tissues with tumor metastasis were analyzed for markers of oxidative stress and inflammation and for HIF-1a using western blotting and real-time PCR (qRT-PCR). The level of reactive oxygen species (ROS) in B16F10 cell was also assessed after N, IH and IH with tempol treatments.

Results: Compared with normoxia, $I \mathrm{H}$ significantly increased the number and weight of mouse lung metastatic colonies. Treatment of B16F10 cells with IH significantly enhanced ROS generation. Lung tissues with tumor metastasis provided evidence of increased oxidative stress, as assessed by p $22^{\text {phox }}$ and SOD mRNA levels and the NRF2 protein level, as well as increased inflammation, as assessed by TNF-a and IL-6 mRNA levels and the NF-KB P65 protein level. HIF-1a protein levels were increased in response to $\mathrm{IH}$ treatment. Tempol, an important antioxidant, ameliorated IHinduced melanoma lung metastasis in mice and reduced oxidative stress and inflammation responses.

Conclusions: These results support the hypothesis that oxidative stress and inflammation responses play an important role in the pathogenesis of OSA-like $\mathrm{IH}$-induced melanoma lung metastasis in mice. Antioxidant intervention provides a novel strategy for the prevention and treatment of cancer in OSA populations.
\end{abstract}

Keywords: Obstructive sleep apnea, Intermittent hypoxia, Lung metastasis, Oxidative stress, Inflammation, Antioxidant tempol

\footnotetext{
*Correspondence: ningwen108@nankai.edu; tjcaojie123@163.com

†Equal contributors

${ }^{2}$ State Key Laboratory of Medicinal Chemical Biology, College of Life

Sciences, Nankai University, Tianjin, China

${ }^{1}$ Respiratory Department, Tianjin Medical University General Hospital, Tianjin,

China
} 


\section{Background}

Obstructive sleep apnea (OSA), a common sleep breathing disorder, is characterized by recurrent upper airway obstruction triggered by complete or partial upper airway collapse during sleep. It can lead to intermittent hypoxia ( $\mathrm{IH})$, sleep fragmentation and daytime sleepiness. OSA affects at least $4-10 \%$ of adults and has been reported as a critical predisposing factor for cardiovascular morbidity [1-4], metabolic disorder [5, 6], hypertension and cognitive dysfunction [7,8]. Currently, several epidemiological studies have demonstrated that cancer progression and cancer-related mortality are accelerated in patients with OSA [9-14]. Therefore, IH is considered to be a major characteristic factor of OSA for promoting tumor invasion and metastasis $[15,16]$. Animal studies also indicate that OSA-like IH enhances the growth, invasion and metastasis of tumors [17-20]. However, the underlying mechanisms for this OSA-like IH-induced tumor metastasis are not completely understood.

Hypoxia is increasingly recognized as an important feature of the intra-tumoral microenvironment, and it can induce the growth and metastasis of tumors via the up-regulation of hypoxia-inducible factor- $1 \alpha$ (HIF-1 $\alpha$ ) [21]. However, in contrast to continuous hypoxia, IH in patients with OSA is a unique physiological state with a phase of post-hypoxic re-oxygenation (ROX). It is characterized by higher frequency, more serious hypoxia and larger variation in blood oxygen saturation. The relationship between OSA-like IH and tumors has been explored in recent years. The hypothesis that OSA-like IH results in the development and progression of tumors has been proposed in several investigations [17, 22-25]. OSA-like IH includes a phase of post-hypoxic ROX, which results in the production of reactive oxygen species (ROS) and increases oxidative stress and inflammation responses. These represent important pathological links between OSA and injuries of multiple organs including the myocardium [26, 27], carotid body [28, 29], adrenal gland [30] and brain [31,32]. Recently, Gutsche et al. showed that $\mathrm{IH}$ increases pro-metastatic gene expression by activating NF- $\mathrm{kB}$ in inflammatory breast cancer cells [25]. Here, we hypothesized that oxidative stress and inflammation responses may play important roles in a mouse model of OSA-like $\mathrm{IH}$-induced tumor metastasis.

4-Hydroxy-2,2,6,6-tetramethylpiperidine- $\mathrm{N}$-oxyl (tempol), a well-known antioxidant, promotes the clearance of ROS and protects mitochondria from oxidative damage. It has been reported that tempol can reduce the atherosclerosis associated with metabolic syndrome by reducing vascular inflammation and repressing NADPH2 oxidase expression [33]. It also exerts neuroprotective effects by reducing superoxide anions and preventing peroxynitrite-associated inflammation [34-36]. Recently, tempol has been confirmed to attenuate apoptotic signals in endothelial cells from IH-exposed rats by decreasing oxidative stress and inflammation injury [37]. Therefore, it is reasonable to hypothesize that tempol may attenuate $\mathrm{IH}$-induced tumor metastasis by decreasing oxidative stress and inflammation responses. This study was designed to investigate the enhancement of OSA-like IH-induced melanoma lung metastasis by oxidative stress and inflammation responses, and to assess the interventional role of the antioxidant tempol.

\section{Methods \\ Animals}

C57BL/6 J male mice with the age of 8 weeks were purchased from Model Animal Research Center of Nanjing University. All mice were housed and cared for in standard cages and provided access to food and water freely at the General Hospital of Tianjin Medical University. The animals, after being injected with melanoma cells, were randomly distributed into three groups $(n=8): \mathrm{N}$ group, IH group and IHT group, which was treated with tempol (Sigma-Aldrich, St. Louis, MO, USA). All animal experimental protocols were approved by the Animal Care and Use Committee at General Hospital of Tianjin Medical University and were performed in accordance with the guidelines outlined by the committee.

\section{Melanoma cell culture}

Murine melanoma B16F10 cells were obtained from the Laboratory of Lung Development and Diseases at Nankai University (Tianjin, China) with the American Type Culture Collection (ATCC; Manassas, VA, USA) as the original source. These cells were cultured in DMEM supplemented with $10 \%$ fetal bovine serum (Gibco, Carlsbad, CA, USA) and antibiotics in a $37^{\circ} \mathrm{C}$ incubator with a humidified atmosphere containing $5 \% \mathrm{CO}_{2}$.

\section{Establishment of the induced melanoma lung metastasis model}

Briefly, the mice were anaesthetized with chloral hydrate (Sangon Biotech, Shanghai, China) and then intravenously injected with $1 \times 10^{5}$ B16F10 cells diluted in $100 \mu \mathrm{L}$ of PBS via tail vein. The number and weight of metastatic colonies per lung were assessed after 3 weeks.

\section{Animal exposure to $\mathrm{IH}$ and tempol administration}

The animal model of $\mathrm{IH}$ was established according to a previously described protocol [38]. The mice were exposed to $\mathrm{IH}$ for $6 \mathrm{~h} /$ day during the light period (10 AM-4 PM) for 21 consecutive days in a specialized plexiglas chamber $(23 \mathrm{~cm} \times 20 \mathrm{~cm} \times 12 \mathrm{~cm}=$ $\left.5520 \mathrm{~cm}^{3} \approx 5.5 \mathrm{~L}\right)$ with 8 mice per cage. The flow of nitrogen $\left(99.99 \% \mathrm{~N}_{2}\right.$, hypoxia phase) or compressed air (air, ROX phase) was modulated by a gas control 
delivery system in the chamber to maintain a nadir oxygen concentration of $5 \%$ and an $\mathrm{IH}$ cycle. The alternating cycle lasted for $2 \mathrm{~min}$, and consisted of hypoxia and ROX phases. Gas flow and $\mathrm{O}_{2}$ concentration were monitored continuously with an $\mathrm{O}_{2}$ analyzer (CY-12C, Meicheng, China). During the hypoxia phase, the $\mathrm{O}_{2}$ concentration in the chamber was decreased to $5 \%$ within $20 \mathrm{~s}$ by infusion of $\mathrm{N}_{2}$ and remained at that concentration for $30 \mathrm{~s}$. During the ROX phase, the $\mathrm{O}_{2}$ concentration was rapidly increased to $21 \%$ within $10 \mathrm{~s}$ by flushing the chamber with compressed clean air, which was then sustained for $60 \mathrm{~s}$. In general, 180 cycles were completed during the exposure period (10 AM-4 PM) every day. Mice exposed to the N condition were used as the control group. Mice in the IHT group received an intraperitoneal injection of $1 \mathrm{ml}$ of $10 \%(w / v)$ tempol per kilogram body weight prior to $\mathrm{IH}$ exposure every day, throughout the whole exposure period of 3 weeks.

\section{Metastasis assessment}

Five lung lobes were carefully separated from the mice in the IH-induced melanoma metastasis model. The number of metastatic colonies per lung was counted after anterior and posterior image acquisition. Metastatic colonies were then separated from the lung lobes, and the weight of the metastases per lung was measured.

\section{RNA isolation and qRT-PCR}

Samples of mouse lung tumor tissue from the three groups $(n=8)$ were homogenized in Trizol. RNA isolation and qRT-PCR analysis were performed as previously described [39]. The expression of $\mathrm{p} 22^{\text {phox }}, \mathrm{SOD}, \mathrm{TNF}-\alpha$ and IL-6 was determined using a SYBR Green Master Mix kit (Roche Diagnostics, Indianapolis, IN, USA). Mouse $\beta$ actin was used as an internal control. Gene expression was quantified relative to the mRNA levels from mouse lung tumor tissue, which was amplified in parallel. The sequences of specific primer pairs are listed in Table 1.

\section{Western blot analysis}

Proteins were extracted from mouse lung tumor tissue according to standard protocols as described previously [40]. Protein concentrations were measured using a BCA assay kit (Pierce, Rockford, IL, USA). The proteins were separated by SDS-PAGE and electroblotted onto

Table 1 Real-Time PCR primers used in this study

\begin{tabular}{|c|c|c|}
\hline Genes & Forward primer 5'-3' & Reverse primer 5'-3' \\
\hline p22 phox & AAGTACCTGACCGCTGTGG & AGGTAGATCACACTGGCAATG \\
\hline$S O D$ & TTATGATGGGCACTGCAAAGAA & TAGAGAACCAGCC \\
\hline TNF- $a$ & CCAAACCAGCCTGACAACTT & TCTAGCATGCTCCACCACTG \\
\hline $\mathbb{I L}-6$ & GTGTAGCACAACTTCCAATTACGAA & GGAATTTCCGCCTCGAGTCT \\
\hline -actin & AGGCCAACCGTGAAAAGATG & AGAGCATAGCCCTCGTAGATGG \\
\hline
\end{tabular}

PVDF membranes (Millipore, Bedford, MA, USA). These membranes were blocked with $5 \%$ skim milk for $1 \mathrm{~h}$ at room temperature and then incubated with primary antibodies at $4{ }^{\circ} \mathrm{C}$ overnight. The primary antibodies, including HIF-1 $\alpha$ (1:200; Novus Biologicals, Littleton, CO, USA), NF-E2-related factor 2 (NRF2) (1:200; ProteinTech Group, Chicago, IL, USA), NF-кB P65 (1:5000; Santa Cruz Biotechnology Inc., Santa Cruz, CA, USA) and $\beta$-actin (1:5000; Santa Cruz Biotechnology Inc., Santa Cruz, CA, USA), were used to probe the target proteins. Goat anti-mouse IgG-HRP or goat antirabbit IgG-HRP (1:5000; Santa Cruz Biotechnology Inc., Santa Cruz, CA, USA) were used as secondary antibodies and were incubated with the membranes for $2 \mathrm{~h}$ at room temperature. Bands were visualized with ECL reagents. The intensity of protein bands on the scanned images was analyzed with a Tanon Gis digital image analytical system. The accumulation of HIF- $1 \alpha$, NRF2 and NF- $\kappa B$ P65 was quantified as the ratio of the band intensities for the target protein and $\beta$-actin.

\section{ROS detection in vitro}

B16F10 cells were seeded in $60 \mathrm{~mm}$ culture dishes and incubated for $12 \mathrm{~h}$ with DMEM supplemented with $10 \%$ fetal bovine serum. After two washes with PBS, the culture medium was replaced with $2 \mathrm{ml}$ DMEM supplemented with $2 \%$ fetal bovine serum. The cells were treated with $\mathrm{N}$, $\mathrm{IH}\left(30 \mathrm{~s} 5 \% \mathrm{O}_{2}-90 \mathrm{~s} 21 \% \mathrm{O}_{2}\right.$ ) and $\mathrm{IH}$ with tempol for $8 \mathrm{~h} /$ $\mathrm{d}$, for $2 \mathrm{~d}$. ROS generation was determined using a Reactive Oxygen Species Assay Kit (Beyotime, China). The cells were washed twice with pre-warmed $\mathrm{PBS}$ and then incubated with DCFH-DA $(10 \mathrm{mM})$ at $37^{\circ} \mathrm{C}$ for $30 \mathrm{~min}$. After two washes with PBS, the cells were collected with $1 \mathrm{ml}$ lysis buffer. Then, the clear supernatant was transferred to a 96-well plate after centrifugation at $12000 \mathrm{rpm}$ for $10 \mathrm{~min}$. Relative fluorescence was read with a BioTek Cytation 5 System (BioTek, Winooski, VT) set at $488 \mathrm{~nm}$ excitation and $525 \mathrm{~nm}$ emission. All assays were repeated in triplicate.

\section{Statistical analysis}

SPSS version 16.0 (SPSS Inc., Chicago, IL) software package was used for statistical analysis. All data are presented as Mean \pm SEM. Comparisons between several groups were analyzed using one-way ANOVA. Difference between experimental and control group was analyzed using Student's t test. The statistically significant difference was considered at $P<0.05$.

\section{Results \\ OSA-like IH promotes melanoma lung metastasis}

The number of lung metastatic melanomas was determined at day 21 after the exposure to $\mathrm{N}, \mathrm{IH}$ and IHT 
conditions. The number of metastatic melanomas per lung in the IH group $(12.8 \pm 1.5)$ was significantly higher than that in the $\mathrm{N}$ group $(4.8 \pm 0.7)$ (Fig. 1a, b) $(P<0.01)$. However, the number of metastatic melanomas per lung in the IHT group $(5.1 \pm 0.8)$ was lower than that in the IH group (Fig. 1a, b) $(P<0.01)$.

In addition, compared to that of the $\mathrm{N}$ group, the weight of lung metastases in the IH group was significantly increased (Fig. 1c) $(P<0.05)$. The weight of lung metastases in the IHT group was decreased when compared with that of the IH group (Fig. 1c) $(P<0.05)$. These findings suggested that OSA-like $\mathrm{IH}$ increased melanoma metastasis in the lung, and this enhancement effect was prevented by pretreatment with the antioxidant tempol (Fig. 1a, b, c).

Increased ROS generation in murine melanoma cells after exposure to OSA-like IH

OSA-like IH can be interrupted by posthypoxic reoxygenation, which results in the generation of $\mathrm{O}_{2}$ free radicals and oxidative stress. In current study, we first investigated the effect of OSA-like $\mathrm{IH}$ exposure on
ROS levels in murine melanoma B16F10 cells. As shown in Fig. 2, we found that compared with normoxia, OSA-like IH markedly increased ROS levels (Fig. 2) $(P<0.05)$. However, the antioxidant tempol effectively diminishes this OSA-like IHinduced ROS production (Fig. 2).

\section{Activated oxidative stress response in melanoma lung} metastasis mouse model after exposure to OSA-like IH ROS generation can activate oxidative stress and inflammation responses. Accordingly, we investigated the roles of oxidative stress and inflammation responses in the mouse model of OSA-like IH-induced tumor metastasis. To assess the activation of the oxidative stress response in the $\mathrm{IH}$-induced lung metastasis mouse model, the mRNA expression levels of SOD and $\mathrm{p} 22^{\text {phox }}$ were evaluated by $\mathrm{qRT}-\mathrm{PCR}$. The transcription factor NF-E2-related factor 2 (NRF2), which is related to oxidative stress, was evaluated by western blotting. The mRNA expression level of SOD in the IH group was significantly decreased compared with that in the $\mathrm{N}$ group (Fig. 3A)

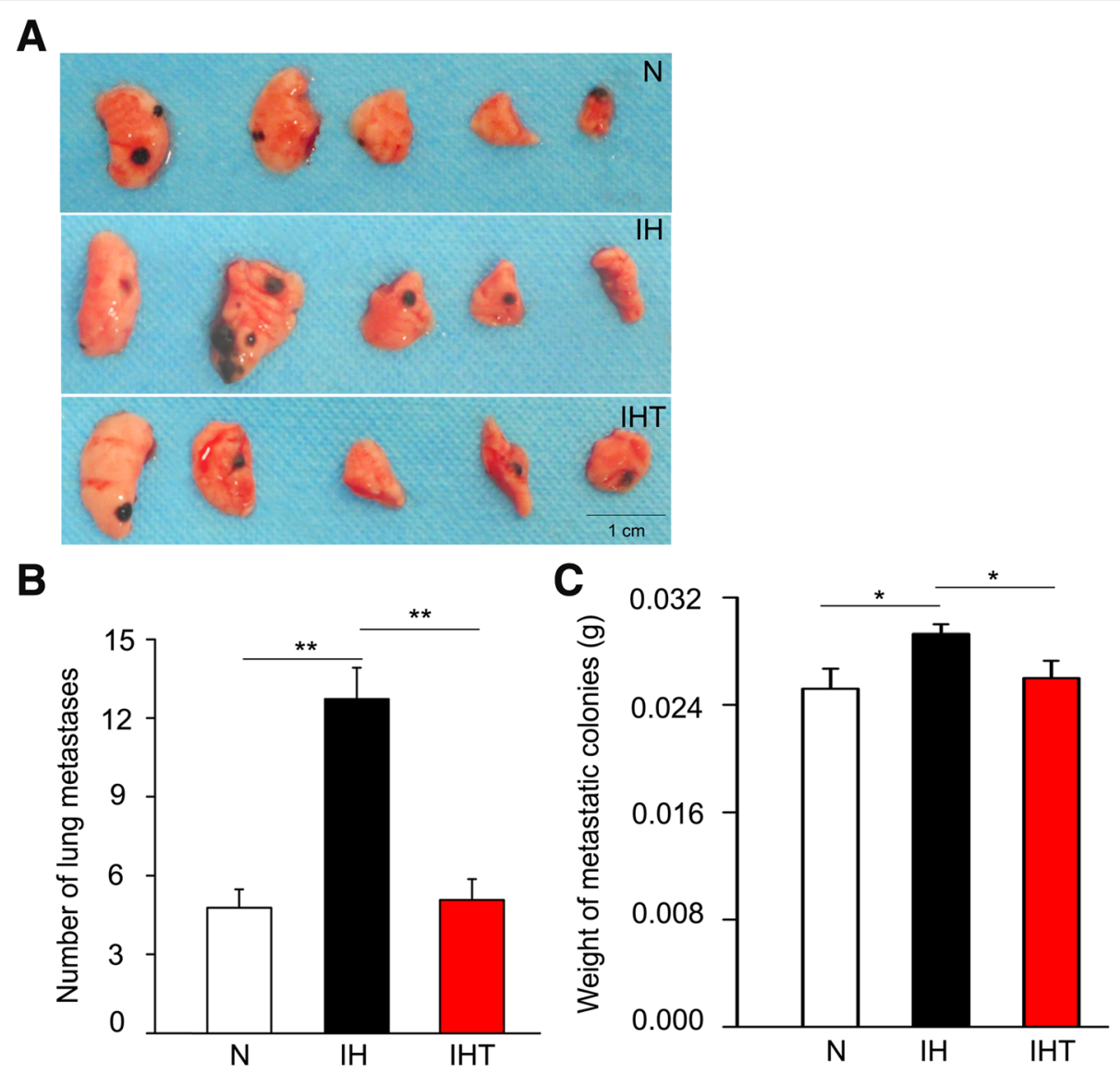

Fig. 1 Effects of OSA-like IH on melanoma lung metastasis. a Example of lung metastasis, visible as black dots on the surface of 5 lung lobes from mice subjected to $\mathrm{N}, \mathrm{IH}$, or $\mathrm{IHT}$ treatment. b Total number of melanoma lung metastatic colonies observed in the $\mathrm{N}, I H$, or $I H T$ groups. ${ }^{* *} P<0.01$. c The weight of melanoma lung metastatic colonies was assessed. ${ }^{*} P<0.05 . n=8$ per group. All data are expressed as the mean \pm SEM 


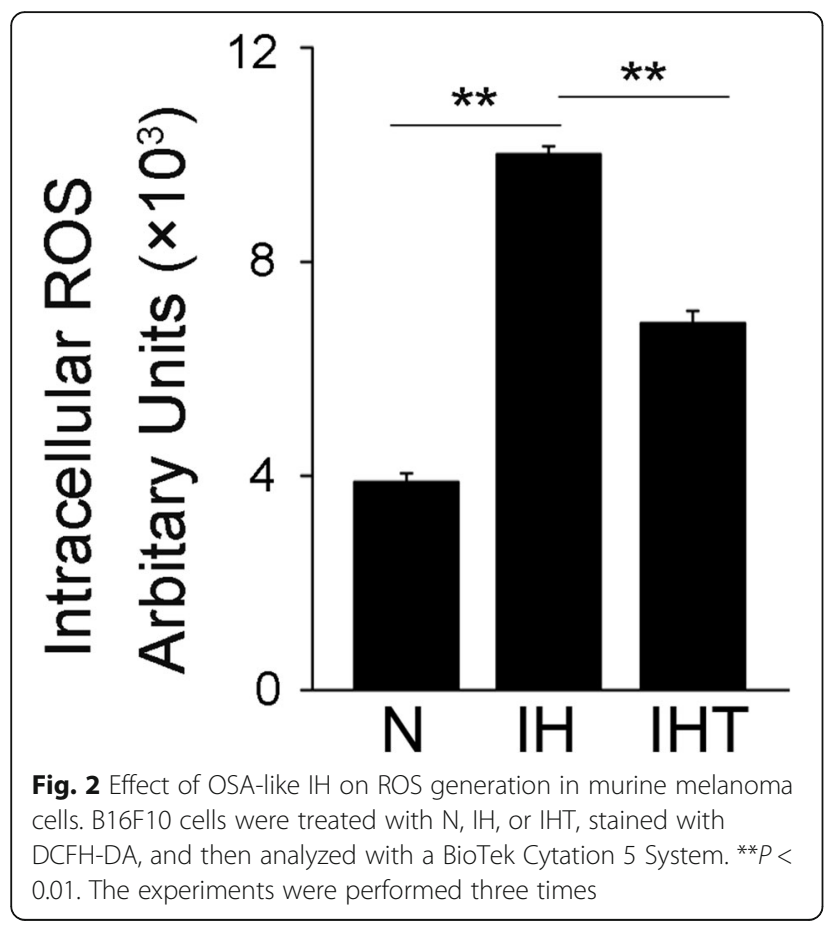

$(P<0.05)$. The mRNA expression level of $\mathrm{p} 22^{\text {phox }}$ in the IH group was also significantly increased compared with that in the $\mathrm{N}$ group (Fig. 3B) $(P<0.05)$. The protein expression level of NRF2 was significantly increased in the $\mathrm{IH}$ group when compared with that in the $\mathrm{N}$ group (Fig. $3 \mathrm{C} \mathrm{a}, \mathrm{b})(P<0.01)$. These results indicated that OSA-like IH enhanced oxidative stress in the melanoma lung metastasis mouse model. As expected, oxidative stress in the IHT group was significantly reduced compared with that in the IH group (Fig. 3A, B, C).

\section{Activated inflammation response in melanoma lung} metastasis mouse model after exposure to OSA-like IH Next, to examine the activation of the inflammation response in the OSA-like $\mathrm{IH}$-induced lung metastasis mouse model, the mRNA expression levels of TNF- $\alpha$ and IL-6, as well as the protein level of the inflammation response transcription factor NF- $\mathrm{kB}$ P65, were evaluated by qRT-PCR and western blotting, respectively. The mRNA expression levels of TNF- $\alpha$ and IL-6 in IH group were significantly increased when compared with those in the $\mathrm{N}$ group (Fig. 4A, B) $(P<0.05)$. The protein expression level of NF- $k$ B $\mathrm{P} 65$ in the IH group was significantly increased when compared with that in the $\mathrm{N}$ group (Fig. $4 \mathrm{C}$ a, b) $(P<0.05)$. These results suggested that OSA-like $\mathrm{IH}$ activated the inflammation response in this melanoma lung metastasis mouse model. Similarly, inflammation in the IHT group was significantly attenuated when compared with that in the IH group (Fig. 4A, B, C).
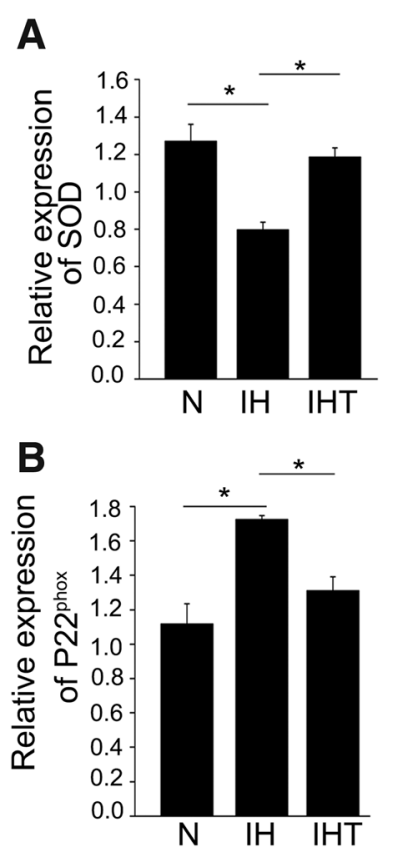

\section{C}

a



b

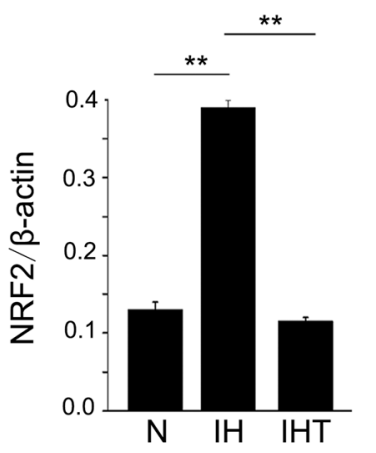

Fig. 3 Effect of OSA-like IH on oxidative stress responses in melanoma lung metastasis mice. qRT-PCR analysis of SOD (A) and p22 $2^{\text {phox }}$ (B) mRNA expression ( $n=6$ per group) and western blotting analysis of NRF2 protein expression $(\mathbf{C} a$ ) in tumor tissue from mouse lungs after OSA-like $1 \mathrm{H}$ exposure and/or tempol treatment ( $n=3$ per group). The expression level of NRF2 was evaluated by densitometric analysis of western blot bands (C b). All data are expressed as the mean \pm SEM. ${ }^{*} P<0.05,{ }^{*} P<0.01$ 



Fig. 4 Effect of OSA-like IH on the inflammatory response in melanoma lung metastasis mice. qRT-PCR analysis of TNF-a (A) and IL-6 (B) mRNA expression ( $n=6$ per group) and western blotting analysis of NF-KB P65 protein expression $(\mathbf{C} a$ ) in tumor tissue from mouse lungs after OSA-like IH exposure and/or tempol treatment ( $n=3$ per group). The expression level of NF-kB P65 (C $b$ ) was evaluated by densitometric analysis of western blot bands. All data are expressed as the mean \pm SEM. ${ }^{*} P<0.05$

Increased HIF-1 1 a expression in melanoma lung metastasis mouse model after exposure to OSA-like IH

HIF- $1 \alpha$ is an important transcription factor for responding to hypoxia, and plays an important role in tumor metastasis. The protein expression level of HIF-1 $\alpha$ in the $\mathrm{IH}$ group was significantly higher than that in the $\mathrm{N}$ group $(P<0.05)$. However, the protein expression level of HIF- $1 \alpha$ in the IHT group was significantly lower than that in the IH group (Fig. 5a, b) $(P<0.05)$, suggesting that tempol treatment attenuates the OSA-like IHinduced expression of HIF- $1 \alpha$ in this melanoma lung metastasis mouse model.

\section{Discussion}

Epidemiological studies have demonstrated that OSA can promote the development and mortality of cancers. Recently, the relationship between OSA-like IH and tumors has only been explored in animals. In the present study, OSA-like IH exposure increased melanoma metastasis in the lung, which is remarkably similar to previous reports [18], consistently with the observation that patients with OSA have a close relationship with high cancer incidence and cancer-related mortality [10-14].

OSA-like IH may be interrupted by ROX. In the ROX phase, excessive ROS, primarily derived from activated NADPH oxidase, can lead to oxidant/antioxidant imbalance, and cause radical-induced oxidation and damage. Many studies have confirmed that oxidative stress is associated with the occurrence and progression of tumors

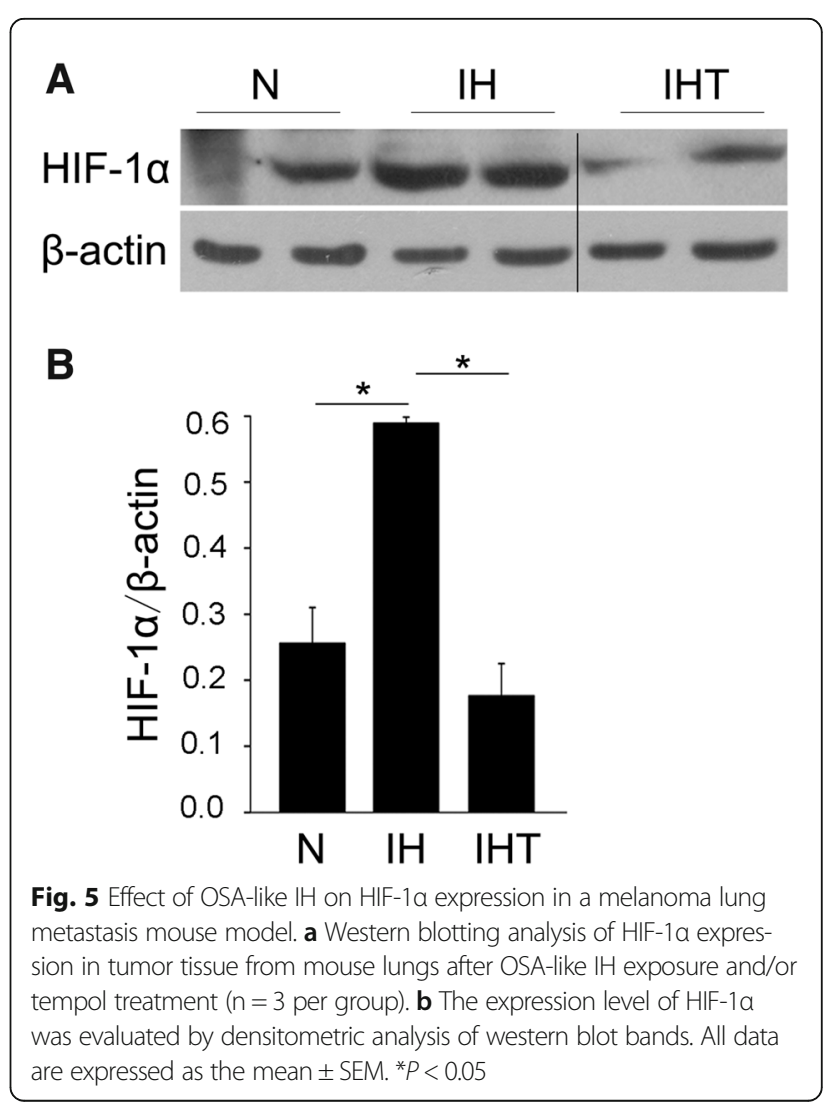


[41]. Herein, we have reported that OSA-like IH enhanced ROS generation in B16F10 cells. The mRNA expression level of the enzymatic antioxidant SOD was significantly lower in the IH group than in the $\mathrm{N}$ group. In addition, the mRNA expression level of NADPH oxidase $\mathrm{p} 22^{\text {phox }}$ in the $\mathrm{IH}$ group was significantly increased when compared with that in the N group. NRF2, which can be activated by $\mathrm{IH}$, is a key transcription factor for responding to oxidative stress. In the OSA-like IHinduced lung metastasis mouse model, the protein expression level of NRF2 in the IH group was significantly elevated compared with that in the $\mathrm{N}$ group. These results suggested that oxidative stress might be the mechanism underlying the melanoma lung metastasis induced by OSA-like IH.

Oxidative stress promotes inflammation responses, and in turn, inflammation enhances oxidative stress. NF- $\mathrm{KB}$ is another transcription factor activated by ROS, and it acts as a master regulator of the transcriptional responses to inflammation [42, 43]. Up-regulation of inflammatory molecules or cytokines is associated with tumor proliferation, invasiveness and metastasis [44, 45]. Gutsche et al. showed that in inflammatory breast cancer cells, IH induced the expression of multiple pro-metastatic genes via NF-kB [25]. In our study, we also verified that OSA-like $\mathrm{IH}$ significantly increased the protein level of NF- $\mathrm{kB}$ in a melanoma lung metastasis mouse model. The mRNA levels of inflammatory markers, including TNF- $\alpha$ and IL6 , were significantly induced by OSA-like IH exposure. Inflammatory responses activated by oxidative stress may be a potential mechanism underlying the melanoma lung metastasis induced by OSA-like IH.

Excessive ROS can be eliminated by exogenous antioxidants in several animal models $[46,47]$. Tempol, a redoxcycling nitroxyl radical, can scavenge excessive ROS and increase SOD activity, and is thus considered to be a promising antioxidant for clinical and experimental application. It exerts neuroprotective effects by reducing superoxide anions and attenuating peroxynitrite-associated inflammation. Previous studies have demonstrated that tempol plays a protective role in cardiovascular disease, neurological disorders and cancer by reducing oxidative stress or inflammation. In our OSA-like IH-induced lung metastasis mouse model, tempol repressed the IHinduced oxidative stress and inflammation responses, as well as melanoma lung metastasis. In the future, antioxidant intervention may be an effective strategy for treating OSA-related cancer patients.

HIF- $1 \alpha$ is an important transcription factor for the adaptive response to hypoxia. HIF- $1 \alpha$ protein accumulates under hypoxic conditions, and is degraded after each reoxygenation [48]. In our study, the protein level of HIF- $1 \alpha$ in the IH group was significantly higher than that in the $\mathrm{N}$ group, which was consistent with previous studies [24, 49,
50]. IH can induce ROS generation and cause oxidative stress responses. Tempol, known as an antioxidant, promotes the clearance of ROS and protects mitochondria from oxidative damage. Previous reports have shown that tempol or other antioxidants can prevent HIF- $1 \alpha-$ mediated cancer progression [37, 51-53]. Consistent with these reports, our findings suggest that antioxidant tempol attenuated melanoma lung metastasis and oxidative stress, as well as decreased HIF- $1 \alpha$ expression. Therefore, our study indicates that increased ROS production and oxidative stress induced by OSA-like IH promote melanoma lung metastasis, which is in part regulated through upregulation of HIF- $1 \alpha$ expression. However, other studies have demonstrated that the stabilization of HIF- $1 \alpha$ under hypoxia or $\mathrm{IH}$ is independent of oxidative stress [25, 54, 55]. These conflicting reports indicate that further study is necessary to confirm the relationship between HIF-1 $\alpha$ and oxidative stress.

\section{Conclusions}

These results suggest that the oxidative stress and inflammation responses induced by $\mathrm{IH}$ exposure play important roles in the pathogenesis of tumor metastasis, and the use of antioxidants such as tempol may potentially serve as a unique strategy for OSA-related cancer patients.

\section{Acknowledgements \\ None. \\ Funding \\ This study was supported by the grants from the National Natural Science Foundation of China (No. 81500070 and 81670084), and grants from Tianjin Medical University General Hospital for Young Scholars (ZYYFY2014011).}

\section{Availability of data and materials}

All data generated and analyzed during the study are included in the published article and can be shared upon request.

\section{Authors' contributions}

$J C, W N$ and $L L$ conducted the design of the experiments; LL, F-YR, CQ, L-QX, $M-L L$ and $Y$-SF performed the experiments; LL, F-YR, JF and B-YC analyzed the data; JC, WN and LL completed the writing of the manuscript. All authors reviewed the manuscript. All authors read and approved the final manuscript.

\section{Ethics approval}

All animal experimental protocols were approved by the Animal Care and Use Committee at General Hospital of Tianjin Medical University and in accordance with the guidelines outlined by the committee.

Consent for publication

Not applicable

\section{Competing interests}

The authors declare that they have no competing interests.

\section{Publisher's Note}

Springer Nature remains neutral with regard to jurisdictional claims in published maps and institutional affiliations. 
Received: 6 June 2017 Accepted: 23 January 2018 Published online: 12 February 2018

\section{References}

1. Bassetti C, Aldrich MS. Sleep apnea in acute cerebrovascular diseases: final report on 128 patients. Sleep. 1999;22:217-23.

2. Drummond MJ, Glynn EL, Fry CS, Dhanani S, Volpi E, Rasmussen BB. Essential amino acids increase microRNA-499, -208b, and -23a and downregulate myostatin and myocyte enhancer factor 2C mRNA expression in human skeletal muscle. J Nutr. 2009;139:2279-84.

3. Somers VK, White DP, Amin R, Abraham WT, Costa F, Culebras A, Daniels S, Floras JS, Hunt CE, Olson L, et al. Sleep apnea and cardiovascular disease: an American Heart Association/American College of Cardiology Foundation scientific statement from the American Heart Association Council for high blood pressure research professional education committee, council on clinical cardiology, stroke council, and council on cardiovascular nursing. J Am Coll Cardiol. 2008;52:686-717

4. Kokturk O, Ciftci TU, Mollarecep E, Ciftci B. Elevated C-reactive protein levels and increased cardiovascular risk in patients with obstructive sleep apnea syndrome. Int Heart J. 2005;46:801-9.

5. Lurie A. Metabolic disorders associated with obstructive sleep apnea in adults. Adv Cardiol. 2011;46:67-138.

6. Parish JM, Adam T, Facchiano L. Relationship of metabolic syndrome and obstructive sleep apnea. J Clin Sleep Med. 2007;3:467-72.

7. Felmet KA, Petersen M. Obstructive sleep apnea and cognitive dysfunction. JAAPA. 2006;19:16-20.

8. Ferini-Strambi L, Baietto C, Di Gioia MR, Castaldi P, Castronovo C, Zucconi M, Cappa SF. Cognitive dysfunction in patients with obstructive sleep apnea (OSA): partial reversibility after continuous positive airway pressure (CPAP). Brain Res Bull. 2003;61:87-92.

9. Cao J, Feng J, Li L, Chen B. Obstructive sleep apnea promotes cancer development and progression: a concise review. Sleep Breath. 2015;19:453-7.

10. Campos-Rodriguez F, Martinez-Garcia MA, Martinez M, Duran-Cantolla J, Pena Mde L, Masdeu MJ, Gonzalez M, Campo F, Gallego I, Marin JM, et al. Association between obstructive sleep apnea and cancer incidence in a large multicenter Spanish cohort. Am J Respir Crit Care Med. 2013;187:99-105.

11. Martinez-Garcia MA, Campos-Rodriguez F, Duran-Cantolla J, de la Pena M, Masdeu MJ, Gonzalez M, Del Campo F, Serra PC, Valero-Sanchez I, Ferrer MJ, et al. Obstructive sleep apnea is associated with cancer mortality in younger patients. Sleep Med. 2014;15:742-8.

12. Nieto FJ, Peppard PE, Young T, Finn L, Hla KM, Farre R. Sleep-disordered breathing and cancer mortality: results from the Wisconsin sleep cohort study. Am J Respir Crit Care Med. 2012;186:190-4.

13. Zhang X, Giovannucci EL, Wu K, Gao X, Hu F, Ogino S, Schernhammer ES, Fuchs CS, Redline S, Willett WC, Ma J. Associations of self-reported sleep duration and snoring with colorectal cancer risk in men and women. Sleep. 2013;36:681-8

14. Christensen AS, Clark A, Salo P, Nymann P, Lange P, Prescott E, Rod NH. Symptoms of sleep disordered breathing and risk of cancer: a prospective cohort study. Sleep. 2013;36:1429-35.

15. Rofstad EK, Gaustad JV, Egeland TA, Mathiesen B, Galappathi K. Tumors exposed to acute cyclic hypoxic stress show enhanced angiogenesis, perfusion and metastatic dissemination. Int J Cancer. 2010;127:1535-46.

16. Lee SL, Rouhi P, Dahl Jensen L, Zhang D, Ji H, Hauptmann G, Ingham P, Cao Y. Hypoxia-induced pathological angiogenesis mediates tumor cell dissemination, invasion, and metastasis in a zebrafish tumor model. Proc Natl Acad Sci U S A. 2009;106:19485-90.

17. Almendros I, Wang Y, Becker L, Lennon FE, Zheng J, Coats BR, Schoenfelt KS, Carreras A, Hakim F, Zhang SX, et al. Intermittent hypoxia-induced changes in tumor-associated macrophages and tumor malignancy in a mouse model of sleep apnea. Am J Respir Crit Care Med. 2014;189:593-601.

18. Almendros I, Montserrat JM, Torres M, Dalmases M, Cabanas ML, CamposRodriguez F, Navajas D, Farre R. Intermittent hypoxia increases melanoma metastasis to the lung in a mouse model of sleep apnea. Respir Physiol Neurobiol. 2013;186:303-7.

19. Almendros I, Montserrat JM, Torres M, Bonsignore MR, Chimenti L, Navajas D, Farre R. Obesity and intermittent hypoxia increase tumor growth in a mouse model of sleep apnea. Sleep Med. 2012;13:1254-60.
20. Almendros I, Montserrat JM, Ramirez J, Torres M, Duran-Cantolla J, Navajas D, Farre R. Intermittent hypoxia enhances cancer progression in a mouse model of sleep apnoea. Eur Respir J. 2012;39:215-7.

21. Pistollato F, Rampazzo E, Persano L, Abbadi S, Frasson C, Denaro L, D'Avella D, Panchision DM, Della Puppa A, Scienza R, Basso G. Interaction of hypoxia-inducible factor-1alpha and notch signaling regulates medulloblastoma precursor proliferation and fate. Stem Cells. 2010;28:1918-29.

22. Almendros I, Gileles-Hillel A, Khalyfa A, Wang Y, Zhang SX, Carreras A, Farre R, Gozal D. Adipose tissue macrophage polarization by intermittent hypoxia in a mouse model of OSA: effect of tumor microenvironment. Cancer Lett. 2015;361:233-9.

23. Cortese R, Almendros I, Wang Y, Gozal D. Tumor circulating DNA profiling in xenografted mice exposed to intermittent hypoxia. Oncotarget. 2015;6:556-69.

24. Liu Y, Song X, Wang X, Wei L, Liu X, Yuan S, LV L. Effect of chronic intermittent hypoxia on biological behavior and hypoxia-associated gene expression in lung cancer cells. J Cell Biochem. 2010;111:554-63.

25. Gutsche K, Randi EB, Blank V, Fink D, Wenger RH, Leo C, Scholz CC. Intermittent hypoxia confers pro-metastatic gene expression selectively through NF-kappaB in inflammatory breast cancer cells. Free Radic Biol Med. 2016;101:129-42.

26. Ding $W$, Zhang $X$, Huang $H$, Ding $N$, Zhang $S$, Hutchinson SZ, Zhang $X$. Adiponectin protects rat myocardium against chronic intermittent hypoxia-induced injury via inhibition of endoplasmic reticulum stress. PLoS One. 2014;9:e94545.

27. Almendros I, Farre R, Torres M, Bonsignore MR, Dalmases M, Ramirez J, Navajas D, Montserrat JM. Early and mid-term effects of obstructive apneas in myocardial injury and inflammation. Sleep Med. 2011;12:1037-40.

28. Del Rio R, Moya EA, Parga MJ, Madrid C, Iturriaga R. Carotid body inflammation and cardiorespiratory alterations in intermittent hypoxia. Eur Respir J. 2012;39:1492-500

29. Lam SY, Liu Y, Ng KM, Lau CF, Liong EC, Tipoe GL, Fung ML. Chronic intermittent hypoxia induces local inflammation of the rat carotid body via functional upregulation of proinflammatory cytokine pathways. Histochem Cell Biol. 2012;137:303-17.

30. Kumar GK, Rai V, Sharma SD, Ramakrishnan DP, Peng YJ, Souvannakitti D, Prabhakar NR. Chronic intermittent hypoxia induces hypoxia-evoked catecholamine efflux in adult rat adrenal medulla via oxidative stress. J Physiol. 2006;575:229-39.

31. Zhan G, Serrano F, Fenik P, Hsu R, Kong L, Pratico D, Klann E, Veasey SC. NADPH oxidase mediates hypersomnolence and brain oxidative injury in a murine model of sleep apnea. Am J Respir Crit Care Med. 2005;172:921-9.

32. Xu W, Chi L, Row BW, Xu R, Ke Y, Xu B, Luo C, Kheirandish L, Gozal D, Liu R. Increased oxidative stress is associated with chronic intermittent hypoxiamediated brain cortical neuronal cell apoptosis in a mouse model of sleep apnea. Neuroscience. 2004;126:313-23.

33. Cannizzo B, Quesada I, Militello R, Amaya C, Miatello R, Cruzado M, Castro C. Tempol attenuates atherosclerosis associated with metabolic syndrome via decreased vascular inflammation and NADPH-2 oxidase expression. Free Radic Res. 2014:48:526-33.

34. Hahn SM, Mitchell JB, Shacter E. Tempol inhibits neutrophil and hydrogen peroxide-mediated DNA damage. Free Radic Biol Med. 1997:23:879-84.

35. Lipman T, Tabakman R, Lazarovici P. Neuroprotective effects of the stable nitroxide compound Tempol on 1-methyl-4-phenylpyridinium ion-induced neurotoxicity in the nerve growth factor-differentiated model of pheochromocytoma PC12 cells. Eur J Pharmacol. 2006;549:50-7.

36. Rak R, Chao DL, Pluta RM, Mitchell JB, Oldfield EH, Watson JC. Neuroprotection by the stable nitroxide Tempol during reperfusion in a rat model of transient focal ischemia. J Neurosurg. 2000:92:646-51.

37. Guo H, Cao J, Li J, Yang X, Jiang J, Feng J, Li S, Zhang J, Chen B. Lymphocytes from intermittent hypoxia-exposed rats increase the apoptotic signals in endothelial cells via oxidative and inflammatory injury in vitro. Sleep Breath. 2015;19:969-76.

38. He Q, Yang QC, Zhou Q, Zhu H, Niu WY, Feng J, Wang Y, Cao J, Chen BY. Effects of varying degrees of intermittent hypoxia on proinflammatory cytokines and adipokines in rats and 3T3-L1 adipocytes. PLoS One. 2014:9:e86326.

39. Dong Y, Geng Y, Li L, Li X, Yan X, Fang Y, Li X, Dong S, Liu X, Li X, et al. Blocking follistatin-like 1 attenuates bleomycin-induced pulmonary fibrosis in mice. J Exp Med. 2015;212:235-52. 
40. Geng Y, Dong Y, Yu M, Zhang L, Yan X, Sun J, Qiao L, Geng H, Nakajima M, Furuichi T, et al. Follistatin-like 1 (Fstl1) is a bone morphogenetic protein (BMP) 4 signaling antagonist in controlling mouse lung development. Proc Natl Acad Sci U S A. 2011;108:7058-63.

41. Reuter S, Gupta SC, Chaturvedi MM, Aggarwal BB. Oxidative stress, inflammation, and cancer: how are they linked? Free Radic Biol Med. 2010; 49:1603-16.

42. Scholz CC, Taylor CT. Hydroxylase-dependent regulation of the NF-kappaB pathway. Biol Chem. 2013;394:479-93.

43. Muller-Edenborn K, Leger K, Glaus Garzon JF, Oertli C, Mirsaidi A, Richards PJ, Rehrauer H, Spielmann P, Hoogewijs D, Borsig L, et al. Hypoxia attenuates the proinflammatory response in colon cancer cells by regulating IkappaB. Oncotarget. 2015;6:20288-301.

44. Germano G, Allavena P, Mantovani A. Cytokines as a key component of cancer-related inflammation. Cytokine. 2008:43:374-9.

45. Mantovani A, Allavena P, Sica A, Balkwill F. Cancer-related inflammation. Nature. 2008;454:436-44.

46. Kita T, Nagano Y, Yokode M, Ishii K, Kume N, Ooshima A, Yoshida H, Kawai C. Probucol prevents the progression of atherosclerosis in Watanabe heritable hyperlipidemic rabbit, an animal model for familial hypercholesterolemia. Proc Natl Acad Sci U S A. 1987;84:5928-31.

47. Williams RJ, Motteram JM, Sharp CH, Gallagher PJ. Dietary vitamin E and the attenuation of early lesion development in modified Watanabe rabbits. Atherosclerosis. 1992;94:153-9.

48. Toffoli S, Michiels C. Intermittent hypoxia is a key regulator of cancer cell and endothelial cell interplay in tumours. FEBS J. 2008:275:2991-3002.

49. Louie E, Nik S, Chen JS, Schmidt M, Song B, Pacson C, Chen XF, Park S, Ju J, Chen El. Identification of a stem-like cell population by exposing metastatic breast cancer cell lines to repetitive cycles of hypoxia and reoxygenation. Breast Cancer Res. 2010;12:R94

50. Yuan G, Nanduri J, Khan S, Semenza GL, Prabhakar NR. Induction of HIF-1alpha expression by intermittent hypoxia: involvement of NADPH oxidase, Ca2+ signaling, prolyl hydroxylases, and mTOR. J Cell Physiol. 2008;217:674-85.

51. Ma J, Zhang Q, Chen S, Fang B, Yang Q, Chen C, Miele L, Sarkar FH, Xia J, Wang Z. Mitochondrial dysfunction promotes breast cancer cell migration and invasion through HIF1alpha accumulation via increased production of reactive oxygen species. PLoS One. 2013;8:e69485.

52. Schroedl C, McClintock DS, Budinger GR, Chandel NS. Hypoxic but not anoxic stabilization of HIF-1alpha requires mitochondrial reactive oxygen species. Am J Physiol Lung Cell Mol Physiol. 2002;283:L922-31.

53. Nazarewicz RR, Dikalova A, Bikineyeva A, Ivanov S, Kirilyuk IA, Grigor'ev IA, Dikalov SI. Does scavenging of mitochondrial superoxide attenuate cancer prosurvival signaling pathways? Antioxid Redox Signal. 2013;19:344-9.

54. Chua YL, Dufour E, Dassa EP, Rustin P, Jacobs HT, Taylor CT, Hagen T. Stabilization of hypoxia-inducible factor-1alpha protein in hypoxia occurs independently of mitochondrial reactive oxygen species production. J Biol Chem. 2010;285:31277-84.

55. Hagen T, Taylor CT, Lam F, Moncada S. Redistribution of intracellular oxygen in hypoxia by nitric oxide: effect on HIF1alpha. Science. 2003;302:1975-8.

\section{Submit your next manuscript to BioMed Central and we will help you at every step:}

- We accept pre-submission inquiries

- Our selector tool helps you to find the most relevant journal

- We provide round the clock customer support

- Convenient online submission

- Thorough peer review

- Inclusion in PubMed and all major indexing services

- Maximum visibility for your research

Submit your manuscript at www.biomedcentral.com/submit

C Biomed Central 